\title{
Análisis estructural de una red social en línea: la red española de Flickr
}

\author{
Por José-Luis Ortega e Isidro-F. Aguillo
}

\begin{abstract}
Resumen: El presente trabajo analiza desde un punto de vista estructural la red de miembros de Flickr que viven o han nacido en España. Con ello se pretende conocer como ha evolucionado, cual es su forma, qué grupos se detectan y qué usos realizan dentro de ella. Para ello se han identificado 663 miembros de Flickr a través de un doble proceso de bola de nieve y a continuación se ha extraído con un crawler la fecha de adscripción, fotos y contactos. Los resultados nos muestran que los usuarios adscritos en 2004 tienden a usar Flickr como un medio de comunicación entre aficionados a la fotografía, mientras que los miembros posteriores la utilizan como una red social en sí misma, usando las fotos como una forma de expresar experiencias, opiniones y gustos personales.
\end{abstract}

Palabras clave: Flickr, Redes sociales en línea, Web 2.0, Análisis de redes sociales.

Title: Structural analysis of an online social network: the Spanish network of Flickr

Abstract: This paper intends, from a structural point of view, to analyse the network of Flickr's members who live or were born in Spain. The aim of the study is to know how the network evolves, what is its shape, what groups are detected and what uses are carried out within the network. We identified 663

Flickr members through a double snowball process and harvested their joining date, photos and contacts with a crawler. The results show that the users who joined Flickr in 2004 use the service as a communication medium among amateur photographers, while the subsequent members utilise Flickr as a real social network, using the photos as a way to express personal experiences, opinions and tastes.

Keywords: Flickr, Online social networks, Web 2.0, Social network analysis.

Ortega, José-Luis; Aguillo, Isidro-F. “Análisis estructural de una red social en línea: la red española de Flickr”. En: El profesional de la información, 2008, noviembre-diciembre, v. 17, n. 6, pp. 603-610.

DOI: 10.3145/epi.2008.nov.03

\section{Introducción}

Desde 2005 la world wide web ha evolucionado hacia una nueva forma de entender las relaciones y servicios que ofrece. En este nuevo paradigma llamado web 2.0 (O'Reilly, 2005), ésta se ha convertido en un medio de creación colaborativa en la que usuarios particulares, de forma libre y gratuita, aportan experiencias personales y contenidos propios. Todo ello ha favorecido la aparición de diversas sedes web cuya principal característica es la interacción de personas en el diseño de contenidos. Así, hemos visto el surgimiento exitoso de redes sociales en línea, etiquetado social y folksonomías, y proyectos globales de colaboración (i. e. Wikipedia).
Las redes sociales en línea o sitios de redes sociales (Boyd; Ellison, 2007) pueden ser definidos como servicios basados en la web que permiten a los usuarios construir un perfil público y ofrecen las herramientas que hacen posible la interacción entre ellos, compartiendo intereses, recursos e información. Diversos estudios han centrado su interés en dichos sitios ya que ofrecen la oportunidad de conocer cómo el medio en línea afecta a las relaciones sociales (Ellison; Steinfield; Lampe, 2007; Lenhart; Madden, 2007), qué diferencias estructurales existen con otras redes a gran escala (Golder; Wilkinson; Huberman, 2007; Kumar; Novak; Tomkins, 2006) o qué contenidos caracterizan a estas redes (Thelwall, 2008). 
En España pocos trabajos se han centrado en su estudio. Sin embargo cabe destacar los realizados sobre los blogs (Torres-Salinas; Cabezas-Clavijo; Delgado-López-Cózar, 2008) o sobre el impacto de este paradigma en las bibliotecas (Margaix-Arnal, 2007) o en los negocios (Gamero, 2007).

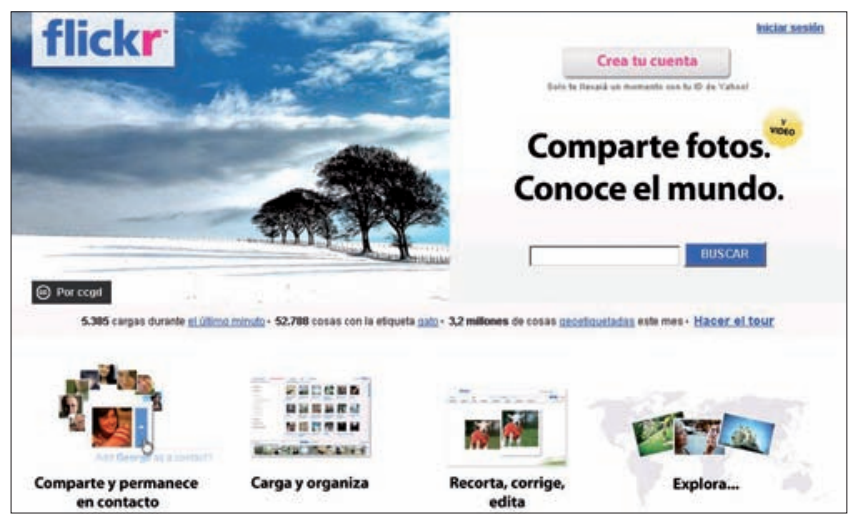

Figura 1. Captura de la web de Flickr

Uno de los primeros productos que representan al mencionado paradigma y que ha sido estudiado desde distintos puntos de vista es Flickr.com. Comenzó en 2003 como un servicio beta, lanzado oficialmente en febrero de 2004 y adquirido por Yahoo! en 2005. Es un repositorio fotográfico que permite subir fotos o imágenes digitales a través de una plataforma sencilla. Junto a las herramientas fotográficas existen utilidades que hacen posible construir perfiles personales, grupos de debate, publicar comentarios y otros servicios propios de las redes sociales. Sin embargo dos características principales han hecho posible su triunfo en la web y su interés para el mundo académico. La primera ha sido el uso de etiquetas (tags) por los usuarios para describir las fotos publicadas, como un sistema de indización social tipo folksonomías (Voss, 2007). Esta característica ha sido estudiada en profundidad (Schmitz, 2006; Angus; Stuart; Thelwall, 2008) debido a que aporta una forma diferente de clasificar e indizar información desde un punto de vista social y podría ser un importante medio de mejora en la recuperación de información visual de grandes repositorios (Kennedy, et al., 2007; Rattenbury; Good; Naaman, 2007).

\section{"Flickr permite a los miembros comentar fotos, crear grupos y construir perfiles, por lo que puede ser una herramienta idónea para el desarrollo de una red social"}

La segunda es la posibilidad de su uso como una plataforma de redes sociales ya que permite a los miembros comentar fotos, crear grupos y construir perfiles. Sin embargo esta característica ha sido menos estudiada y sólo unos cuantos trabajos han tratado este tema. Kumar, Novak y Tomkins (2006) encontraron que existían tres componentes: usuarios solitarios (singletons) que no participan en la red; comunidades aisladas formadas por un pequeño número de socios; y un enorme componente muy conectado. Tanto Prieur et al. (2008) como Pissard y Prieur (2007), usando análisis de componentes principales, se percataron de que podría haber miembros que usasen Flickr como una red social y otros como un repositorio fotográfico. Consideramos interesante citar el trabajo realizado por un miembro de Flickr (Gustavo, 2005), el cual visualizó una red de los 23.000 usuarios más activos, detectando distintos grupos temáticos y geográficos.

\section{Metodología}

Una muestra aleatoria de 59.969 miembros fue seleccionada de Flickr.com en marzo de 2007. Para la extracción se realizaron 33 consultas, preguntando por los números (9) y letras (24) del alfabeto latino. Después de eliminar duplicados, se obtuvieron 23.000 miembros. De este primer grupo sólo 5.800 (25\%) tenían algún contacto y únicamente 400 estaban interconectados $(1,74 \%)$. Esto nos obligó a elegir una muestra mayor para poder contar con más elementos interconectados. Así, de este grupo de 23.000, hemos ampliado la muestra a través de dos procesos de bola de nieve (snowball sampling). Esta técnica de muestreo es muy apropiada para la obtención de muestras en el ámbito de las redes sociales. Consiste en realizar ampliaciones añadiendo los contactos externos a la muestra de los miembros seleccionados hasta conseguir llegar a toda la población o a una cantidad representativa (Biernacki; Waldorf, 1981). Al final se obtuvieron 114.027 miembros, de los cuales $59.969(52,59 \%)$ estaban interconectados. Por razones de cálculo y computación consideramos esta muestra como representativa de la población total de Flickr. Se extrajeron para este trabajo todos los miembros que vivían o habían nacido en España, resultando un total de 663, un 1,11\% de toda la red. Pese a este bajo porcentaje, España es el noveno país representado (los principales son EUA, con 22,75\%, y Reino Unido, con $5,16 \%$.

Una vez que los integrantes fueron individualizados se obtuvieron distintos atributos de los mismos. Es obvio decir que en ningún momento de este estudio se hará mención particular de ninguno en concreto, para salvaguardar su intimidad. Para cada uno se obtuvo el número total de contactos, cantidad de fotos, nacionalidad, sexo, si son usuarios avanzados (pro) y la fecha 
en que se unieron a la red. El proceso de extracción de datos se realizó a través de una rutina en $S Q L$. El procesamiento y análisis fueron realizados con el paquete estadístico SPSS 15.

\section{"España con un 1,11\% de usuarios, es el noveno país representado en la red, compuesta principalmente por miembros de EUA $(22,75 \%)$ y Reino Unido $(5,16 \%)$ "}

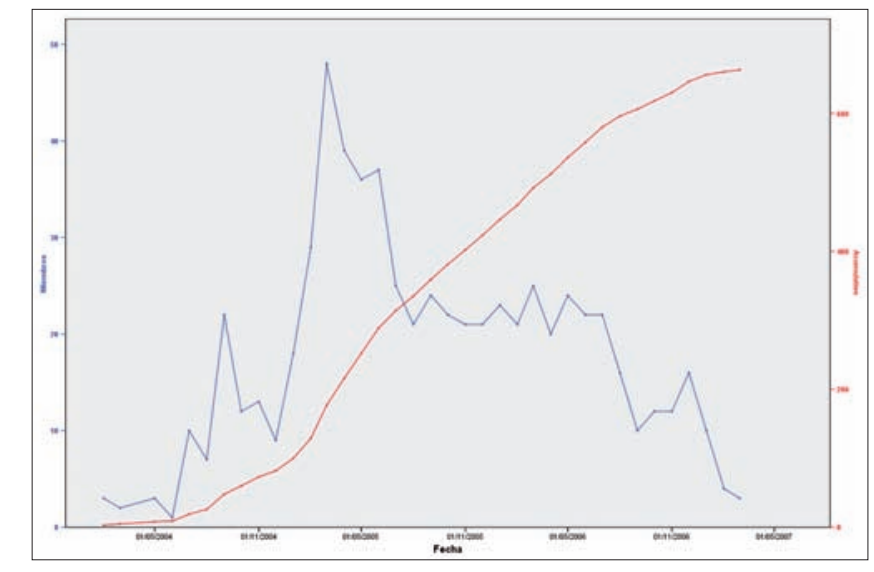

Figura 2. Distribución longitudinal de agregación de nuevos miembros y su curva acumulativa

Dicha red de miembros de Flickr se modelizó con los programas de análisis de redes Pajek 2.01 y Ucinet 6 , que permiten analizar y operar con redes, extrayendo indicadores sobre las principales características de éstas y de los elementos que las componen. Para energizar la red se utilizó el algoritmo de Fruchterman-Reingold, debido a que posee una mayor velocidad en la organización de los nodos en redes de gran tamaño (de Nooy; Mrvar; Batagelj, 2005).

Existen distintas limitaciones en este trabajo debidas principalmente a las características propias del servicio. Una de ellas la encontramos a la hora de recuperar los miembros inscritos en la red. Los resultados en las consultas realizadas en Flickr, son ordenados de acuerdo con la fecha en que se apuntaron. Sin embargo sólo se pueden recuperar los 1.000 primeros en cada consulta. Esto ocasiona que los usuarios más recientes no pueden ser recuperados en algunos casos, obteniendo una población sesgada hacia los más veteranos en la red.

Otra limitación a tener en cuenta es que conocemos cuándo se ha unido un miembro, pero desconocemos en qué momento establece los vínculos con otros. Así, no sabemos cuándo un usuario ha sido contactado por otro. Esto puede distorsionar la percepción evolutiva de la red.

\section{Resultados}

La figura 2 muestra la distribución en el tiempo del número de usuarios españoles que se han ido uniendo a la red, además de su acumulado. En ella se aprecia en un primer momento un incremento constante del número de nuevos miembros hasta un punto de máxima adscripción a mediados de 2005. A continuación desciende hasta una media de 20 usuarios al mes hasta mediados de 2006, en la que el número de los nuevos empieza a descender. Aunque sigue un patrón parecido a la muestra global (Ortega; Aguillo, en prensa), la red española presenta algunas características propias.
El momento de máxima agregación coincide con la fecha en la que Yahoo! adquirió este servicio (febrero de 2005), mientras que en la red global este punto máximo lo encontramos en torno a febrero de 2004, momento en que se lanzó oficialmente el servicio (Ortega; Aguillo, en prensa). También se aprecia que la frecuencia de las nuevas incorporaciones decae a mediados de 2006. Esto puede deberse a las limitaciones mencionadas en la metodología.

Desde un punto de vista estructural, la red muestra propiedades de mundo pequeño (small-world network), caracterizadas porque la distancia media entre nodos es bastante pequeña en relación con el tamaño de la red. Esto hace que su coeficiente de agrupación (probabilidad media de que los nodos formen un grafo completo con sus vecinos) sea más elevado que en las redes aleatorias. Así en nuestro ejemplo, el coeficiente de agrupación $(C C=0,282)$ es mucho mayor de lo esperado para una red aleatoria $(C C=0,005)$ y además posee una distancia media relativamente pequeña de 2,8 clics_Watts; Strogatz, 1998). Sin embargo, el pequeño tamaño de la muestra no nos permite apreciar si estamos también ante una red de libre escala (scalefree network) (Barabasi; Albert; Jeong, 2000), donde la distribución de sus elementos estructurales (grado entrante y saliente) siguen una distribución potencial. Estudios previos sí han certificado sus propiedades en muestras de mayor tamaño (Kumar; Novak; Tomkins, 2006; Prieur et al., 2008; Ortega; Aguillo, en prensa), aunque también han señalado la existencia de propiedades características de redes sociales no comunes a otras de libre escala.

La figura 3 muestra la red española de Flickr. Los colores expresan la fecha en que se adhirieron al servicio y el volumen de los nodos muestra el número de contactos que poseen en la red total de Flickr. Se puede apreciar que los miembros más antiguos poseen menos contactos que los recientes, tal y como vemos también en la tabla 1. Esto puede ser contradictorio, ya 


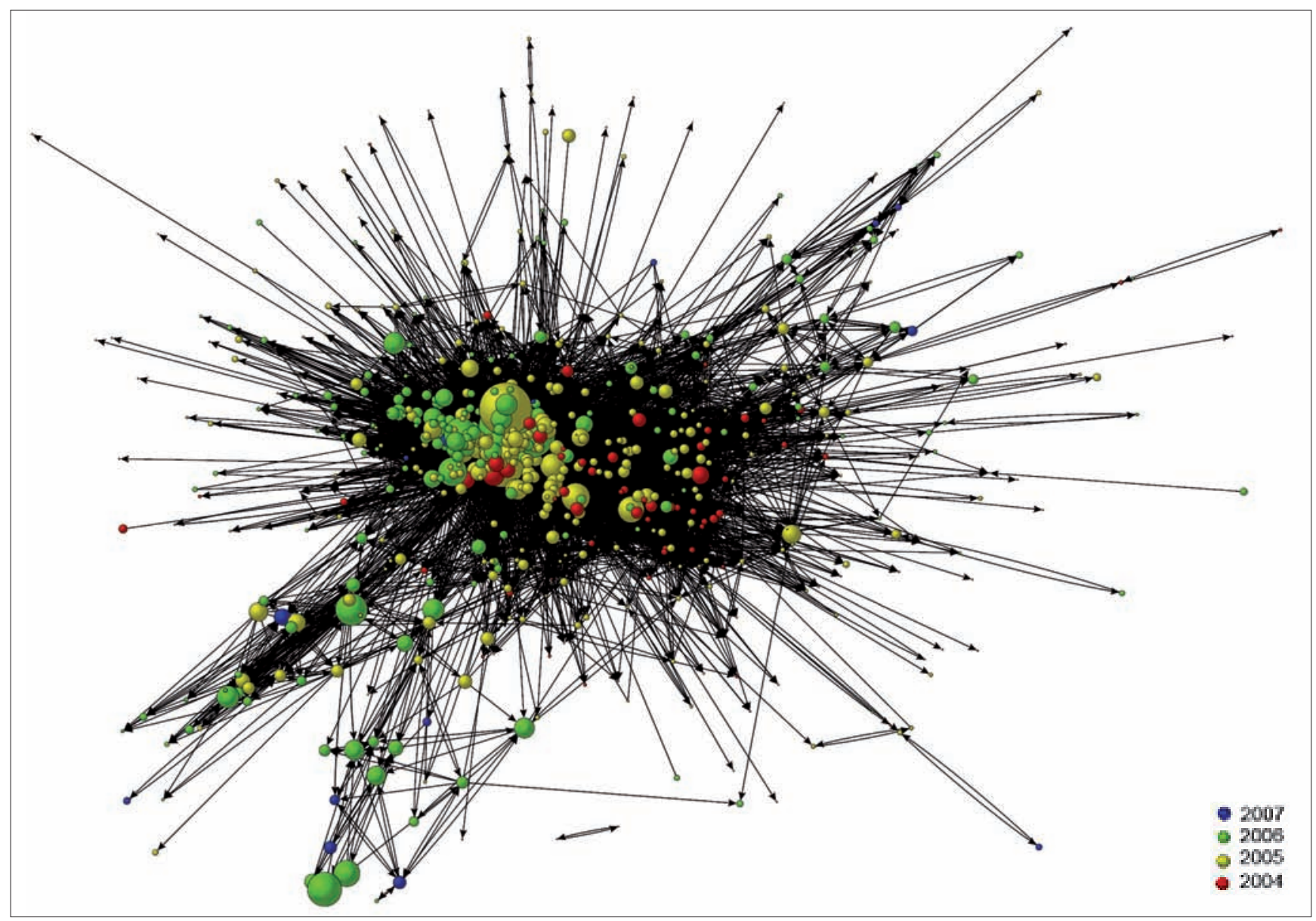

Figura 3. Red Flickr española ( $N=663$; tamaño de los nodos $=n$ o de contactos)

\begin{tabular}{|c|c|c|c|}
\hline Año & Contactos & Pro & \multicolumn{1}{|c|}{ Fotos } \\
\hline 2004 & 212,16 & $62 \%$ & $1.120,43$ \\
\hline 2005 & 300,16 & $62 \%$ & 676,08 \\
\hline 2006 & 392,16 & $52 \%$ & 341,35 \\
\hline 2007 & 213,53 & $41 \%$ & 108,30 \\
\hline
\end{tabular}

Tabla 1. Distribución promedio de contactos, porcentaje de miembros pro y promedio de fotos de los miembros de Flickr según su fecha de adscripción.

que los usuarios antiguos han tenido más tiempo para hacer contactos. Aunque también es cierto que los más recientes poseen más usuarios a quienes enlazar, ya que la red cada vez está más poblada. Sin embargo, la tabla 1 nos sugiere la existencia de diferencias entre los distintos grupos que se unieron a Flickr en cada fecha. Así se aprecia que los usuarios de 2004 hacen muy pocos contactos pero una gran cantidad de fotos, mientras que los usuarios de 2006 casi duplican el número de contactos pero aportan casi un tercio menos de fotos que los de 2004. El bajo número de 2007 en contactos se debe a que sólo son considerados en este estudio los meses de enero y febrero.

El test H de Kruskall-Wallis (1952) nos permite detectar si $n$ grupos de datos pertenecen o no a la mis- ma población. Esta prueba es un estadístico no paramétrico, idóneo para distribuciones no normales como las que encontramos en las redes sociales en línea (Adamic; Buyukkokten; Adar, 2003; Ahn et al., 2007). El resultado de esta prueba nos confirma diferencias en las agrupaciones en cuanto a las fotos que publican ( $p$-valor $<0,0001 ; \alpha=0,05)$, sin embargo no detecta diferencias en cuanto al número de contactos ( $p$-valor $=0,062$; $\alpha=0,05)$. Aunque como vemos, al $90 \%(\alpha=0,1)$ sí existen diferencias entre los grupos. Esto nos permite afirmar que el perfil de los usuarios que se unieron en 2004 es diferente de los que se han unido en 2005 y 2006. La distribución de miembros por fecha en la figura 3 corrobora esta afirmación al mostrar cierta agrupación por fechas, y que los miembros de 2004 se encuentran más dispersos.

"El núcleo de la red ha sido ocupado por miembros de 2005 y 2006, mientras que los primeros miembros de 2004 han quedado relegados a una posición más periférica" 
Utilizando el análisis de k-cores (Seidman, 1983; Ortega; Aguillo, 2007) hemos detectado un núcleo central de 90 nodos (14\%) interconectados con un grado 27. Este grupo está formado principalmente por nodos de 2005 (49\%) y 2006 (36\%), por lo que es evidente que el núcleo de la red ha sido ocupado por miembros de 2005 y 2006, mientras que los primeros miembros de 2004 han quedado relegados a una posición más periférica.

Respecto a la periferia se aprecia la formación de ciertas agrupaciones aisladas de miembros, lo que hace suponer la existencia de algunas comunidades fuertemente vinculadas entre sí. Algunos de ellos poseen un número elevado de contactos, por lo que se deduce que forman parte de una red más amplia e internacional.

En la figura 4 se muestra el mismo gráfico que el anterior (figura 3) pero el tamaño de los nodos expresa esta vez el número de fotografías que posee cada uno. En este caso se aprecia un patrón diferente. Como hemos visto en la tabla 1, los miembros que se unieron en 2004 son los que más fotos publican a la vez que se encuentran más alejados de un centro dominado por miembros de 2005 y 2006.

A fin de descubrir las relaciones que se establecen entre los miembros y de detectar agrupaciones de per- sonas en función de algún criterio, se han calculado los cliques existentes dentro de la red. Un clique puede definirse como una sub-red en la que todos sus nodos están conectados entre sí. La técnica p-cliques (Luce; Perry, 1949; Anderson, 1970) permite detectar estos grupos a partir de un nivel $p$ de restricción. En nuestro estudio se han identificado 206 agrupaciones al nivel de $p<0,05$, de los cuales 7 grupos o cliques principales que representan el $58 \%$ de la red han sido seleccionados por su tamaño. La tabla 2 muestra las características de estos grupos.

Según se aprecia en la figura 5 y la tabla 2, los grupos se caracterizan más por el uso que estos miembros hacen de Flickr, que por afinidades temáticas. Así el grupo mayoritario rojo está formado por miembros que entienden el sitio fundamentalmente como una red social, y en donde las relaciones con otros usuarios son más importantes que subir fotos. Por otro lado, el grupo amarillo está formado por fotógrafos amateur o algunos profesionales que utilizan Flickr como una plataforma para darse a conocer: publica muchas fotografías pero no abunda en las relaciones sociales, siendo éstas muy selectivas. Estos dos grupos suponen el núcleo de la red Flickr española, junto con otros pequeños como el rosa y el azul celeste que poseen ciertas particularidades

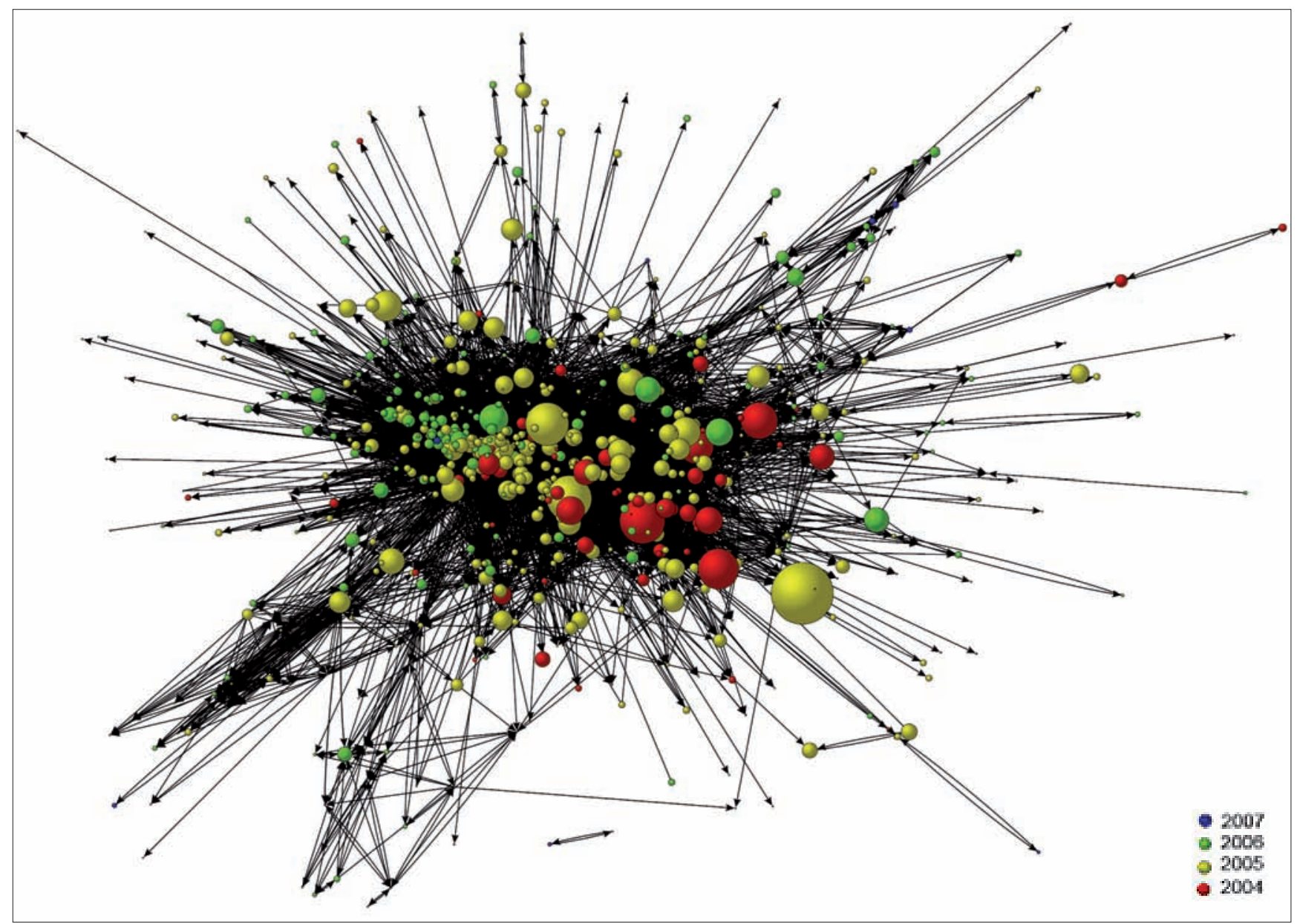

Figura 4. Red Flickr española ( $N=663$; tamaño de los nodos $=n$ o de fotos) 


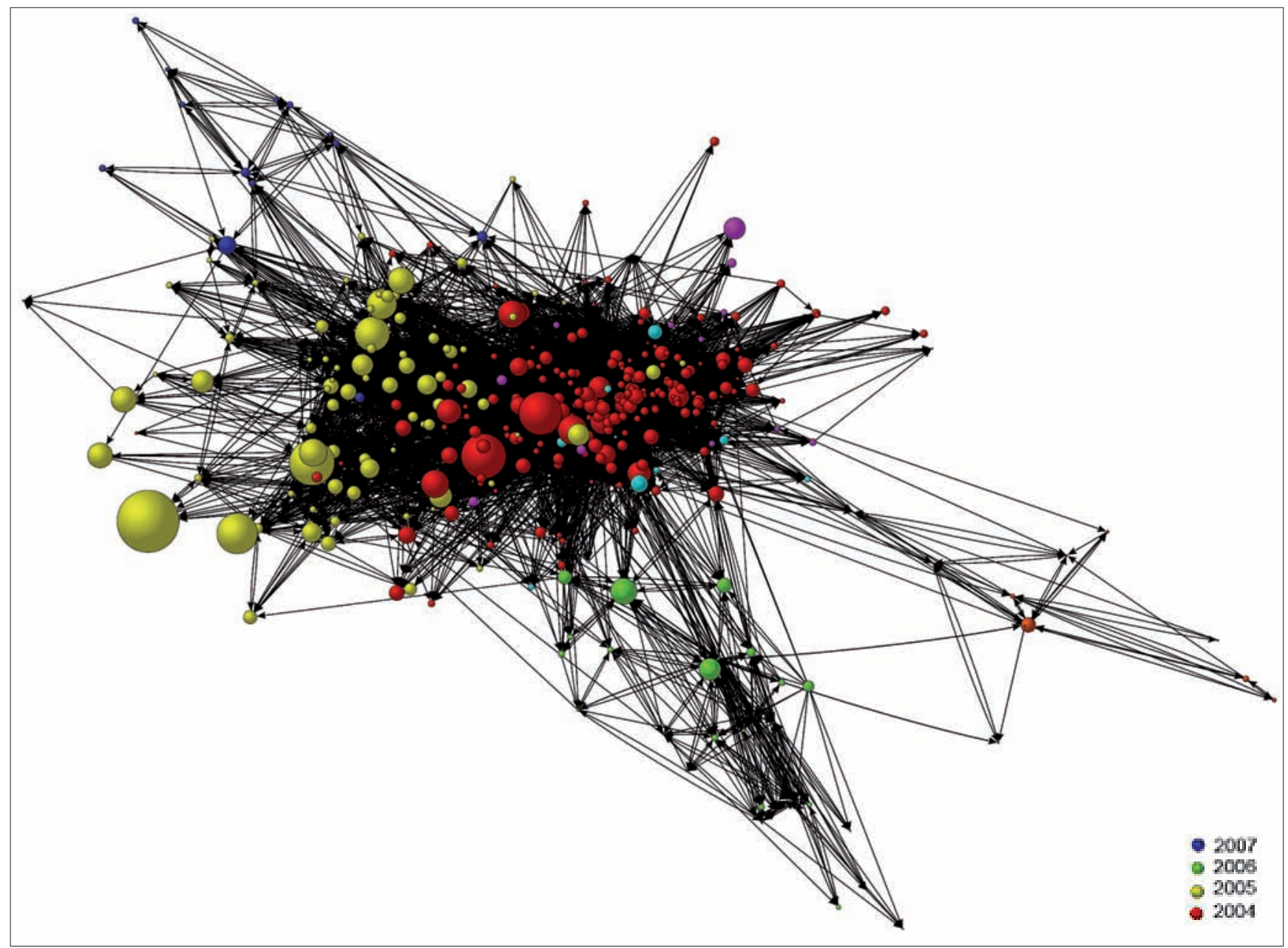

Figura 5. p-cliques de la red Flickr española ( $N=385$; tamaño de los nodos $=n$ ㅇ de fotos)

\begin{tabular}{|c|c|c|c|}
\hline Grupos & Color & Temas & $\begin{array}{c}\text { Miem- } \\
\text { bros }\end{array}$ \\
\hline 6 & Rojo & Genérico & 215 \\
\hline 5 & Amarillo & $\begin{array}{c}\text { Fotografía, } \\
\text { profesional }\end{array}$ & 91 \\
\hline 3 & Verde & Gays & 29 \\
\hline 7 & Azul & Creaciones & 14 \\
\hline 2 & Rosa & $\begin{array}{c}\text { Viajes, } \\
\text { retratos }\end{array}$ & 13 \\
\hline 4 & $\begin{array}{c}\text { Azul } \\
\text { celeste }\end{array}$ & Viajes & 12 \\
\hline 1 & Naranja & Erótico & 11 \\
\hline
\end{tabular}

Tabla 2. Grupos de miembros identificados por la técnica p-cliques $(p<0,05)$

temáticas. Los restantes destacan por su aislamiento y su cohesión interna. Así, el representado por el verde constituye una sub-red propia, aislada del resto pero, como se percibe en la figura 3, parece un apéndice de una red más internacional ya que posee muchos contactos. Al igual que el anterior, el grupo naranja está aislado y publica pocas fotos pero sí tiene una fuerte actividad social, usando el sistema como una web de contactos. Por último, el grupo azul está formado por usuarios que usan Flickr como un medio para dar a conocer sus creaciones como ilustraciones, graffitis, muñecos, etc., a modo de catálogo de productos artesanales puestos a la venta.

"El grupo mayoritario está formado por miembros que entienden Flickr como una red social, donde las relaciones con otros usuarios son más importantes que subir fotos"

\section{Discusión y conclusiones}

La adscripción en el tiempo de nuevos miembros nos permite afirmar que sigue una tendencia lineal. A diferencia de otros elementos en la web como las pá- 
ginas o enlaces (Nielsen, 2007), las redes sociales en línea crecen a un ritmo más pausado (Barabási et al., 2002), debido quizás a que la incorporación de nuevos integrantes es un fenómeno voluntario. Esto implica un mayor esfuerzo por parte de los sitios de redes sociales para crecer que el resto de sitios web, donde la producción de contenidos depende por entero de la organización que los crea. Con respecto al Flickr español, debemos señalar que los usuarios españoles se incorporaron tarde, ya que la gran llegada de miembros no se produce hasta primeros de 2005 cuando Yahoo! la adquirió. En cambio en la muestra mundial esta fecha se adelanta un año (febrero 2004), cuando fue su lanzamiento oficial (Ortega; Aguillo, en prensa). Esto nos hace suponer que hasta la compra de Yahoo! esta red podía tener un carácter local, dominado principalmente por norteamericanos.

\section{"Mientras que los usuarios veteranos utilizaron Flickr como un repositorio fotográfico, los más recientes valoran especialmente la capacidad comunicativa"}

Uno de los resultados más relevantes es la detección de usuarios que actúan de forma diferente en función de la fecha de adscripción al servicio. El test de Kruskal-Wallis nos ha permitido confirmar este hecho. Los miembros que se unieron en los primeros años utilizaron Flickr como un repositorio fotográfico propio de fotógrafos aficionados, los cuales publican gran cantidad de fotos y mantienen pocos contactos. Mientras, las oleadas posteriores de participantes han valorado más la capacidad comunicativa, centrándose en la creación de contactos, y adaptando el uso de fotografías como argumento de comunicación (fotografías personales, eventos, viajes) y no como elementos creativos en sí mismos. Esta diferencia en el uso del servicio es también apreciada por Prieur et al., (2008), que a través de un análisis de componentes principales han apreciado la falta de correlación entre las variables propias de la fotografía (número de fotos y de favoritos) y las variables sociales (número de contactos). Otros estudios han detectado la ausencia de relación entre las fotos que un usuario publica y los contactos que posee (Marlon et al., 2006). Pero más aún, esta diferencia en el uso de la red puede deberse también a un factor local o cultural. Ortega y Aguillo (en prensa) detectaron que los miembros del sudeste asiático publicaban de media más fotos que el resto de los usuarios y al contrario, los de países árabes apenas publicaban fotos, mientras que sus redes de contactos eran más densas que la media restante. Nuestro trabajo introduce otra variable a tener en cuenta: la fecha de adscripción. De esta forma podemos afirmar, al menos para el caso español, que para los usuarios más antiguos, Flickr es un medio para darse a conocer y para interactuar con aficionados con la fotografía, mientras que para los más recientes, es una red social en sí misma y las fotografías son instrumentos para intercambiar experiencias.

Respecto al análisis estructural podemos concluir que la red descansa sobre los miembros más recientes, que priorizan la faceta social del servicio, relegando a un segundo plano a los aficionados a la fotografía. Esto es debido a que los recientes son usuarios más activos en entablar contactos, por lo que ocupan las posiciones más centrales de la red. Así queda claro que la sección española está dominada más por usuarios "sociales" que "fotógrafos". Por otro lado, también se han detectado grupos aislados caracterizados por el uso de temas específicos (erótico, viajes) y usos diferentes y concretos de la red (gays, creaciones). Esto nos muestra los múltiples y diferentes usos que se pueden hacer de Flickr.

Por último, sugerir que el uso de herramientas y técnicas cibermétricas pueden aportar importantes resultados a la investigación de las redes sociales en línea, ya sean entendidas como un fenómeno web o como una extensión del mundo real. Su capacidad para la extracción masiva de datos y el uso de técnicas cuantitativas avanzadas permite mostrar patrones y características de estos nuevos entornos sociales.

\section{Bibliografía}

Adamic, L. A.; Buyukkokten, O.; Adar, E. "A social network caught in the Web". En: First monday, 2003, v. 8, n. 6.

http://www.firstmonday.org/Issues/issue8_6/adamic/index.html

Ahn, Y. Y.; Han, S.; Kwak, H.; Moon, S.; Jeong, H. "Analysis of topological characteristics of huge online social networking services". En: Williamson, C. L.; Zurko, M. E.; Patel-Schneider, P. F.; Shenoy, P. J. (Eds.). Proceedings of the 16th international conference on world wide web, 2007, pp. 835-844. Banff, Canada: ACM Press.

Anderson, S. S. Graph theory and finite combinatorics. Chicago: Markham Publishing Company, 1970.

Angus, E.; Stuart D.; Thelwall, M. "General patterns of tag usage in Flickr image groups". En: Online information review, 2008, v. 32, n. 1.

Barabasi, A. L.; Albert, R.; Jeong, H. "Scale-free characteristics of random networks: the topology of the world-wide web". En: Physica A: statistical mechanics and its applications, 2000, v. 281, n. 1-4, pp. 69-77.

Barabasi, A. L.; Jeong, H.; Neda, Z.; Ravasz, E.; Schubert, A.; Vicsek, T. "Evolution of the social network of scientific collaborations". En: Physica A: statistical mechanics and its applications, 2002, v. 311, n. 3-4, pp. 590614.

Biernacki, P.; Waldorf, D. "Snowball sampling: problems and techniques of chain referral sampling". En: Sociological methods and research, 1981, v. 10, n. 2, pp. 141-163.

Boyd, D. M.; Ellison, N. B. "Social network sites: Definition, history, and scholarship". En: Journal of computer-mediated communication, 2007, v. 13, n. 1, art. 11 .

http://jcmc.indiana.edu/vol13/issue1/boyd.ellison.html 
Ellison, N.; Steinfield, C.; Lampe, C. "The benefits of Facebook 'friends': exploring the relationship between college students' use of online social networks and social capital". En: Journal of computer-mediated communication, 2007, v. 12, n. 3, art. 1

http://jcmc.indiana.edu/vol12/issue4/ellison.html

Gamero, R. "Servicios basados en redes sociales, la web 2.0”. En: Investigación y marketing, 2007, v. 97, pp. 16-20.

Golder, S. A.; Wilkinson, D.; Huberman, B. A. "Rhythms of social interaction: messaging within a massive online network". En: Steinfield, C. Pentland, B.; Ackerman, M.; Contractor, N. (eds.). Proceedings of third international conference on communities and technologies, 2007, pp. 41-66. London: Springer.

Gustavo, G. The FlickrVerse, April 2005 poster: flickr's social network, 2005. Consultado en: 27-05-08.

http://www.flickr.com/photos/gustavog/9708628/

Kennedy, L.; Naaman, M.; Ahern, S.; Nair, R.; Rattenbury, T. "How Flickr helps us make sense of the world: context and content in communitycontributed media collections". En: Lienhart, R.; Prasad, A. R.; Hanjalic, A.; Choi, S.; Bailey, B. P.; Sebe, N. (eds.). Proceedings of the $15^{\text {th }}$ ACM International conference on multimedia (ACM MM 2007), 2007, pp. 631640. Augsburg, Germany: ACM Press.

Kruskal, W. H.; Wallis, W. A. "Use of ranks in one-criterion variance analysis". En: Journal of the American Statistical Association, 1952, v. 47, n. 260, pp. 583-621

Kumar, R.; Novak, J.; Tomkins, A. "Structure and evolution of online social networks". En: Eliassi-Rad, T.; Ungar, L. H.; Craven, M.; Gunopulos, D. (eds.). Proceedings of the 12th ACM Sig kdd international conference on knowledge discovery and data mining, 2006, pp. 611-617. Philadelphia, PA, EUA: ACM Press.

Lenhart, A.; Madden, M. Teens, privacy \& online social networks, 2007. Consultado en: 27-05-08.

http://www.pewinternet.org/pdfs/PIP_Teens_Privacy_SNS_Report_Final. $p d f$

Luce, R.; Perry, A. "A method of matrix analysis of group structure". En: Psychometrika, 1949, v. 14, pp. 95-116.

Marlow, C.; Naaman, M.; Boyd, D.; Davis, M. "HT06, tagging paper, taxonomy, flickr, academic article, toread". En: Wiil, U. K.; Nürnberg, P. J. Rubart, J. (eds.). Proceedings of hypertext 2006, 2006, pp. 31-40. Odense, Denmark: ACM Press.

Margaix-Arnal, D. "Conceptos de web 2.0 y biblioteca 2.0: origen, definiciones y retos para las bibliotecas actuales". En: El profesional de la información, 2007, v. 16, n. 2, pp. 95-106.

Nielsen, J. 100 million websites, 2007. Consultado en: 27-05-08. http://www.useit.com/alertbox/web-growth.html

de Nooy, W.; Mrvar, A.; Batagelj, V. Exploratory social network analysis with Pajek. Cambridge, UK: Cambridge University Press, 2005.

O'Reilly, T. What is web 2.0: design patterns and business models for the next generation of software, 2005. Consultado en: 27-05-08.

http://www.oreillynet.com/pub/a/oreilly/tim/news/2005/09/30/what-is-web20.html

Ortega, J. L.; Aguillo, I. F. "La web académica española en el contexto del Espacio Europeo de Educación Superior: estudio exploratorio”. En: El profesional de la información, 2007, v. 16, n. 5, pp. 417-425.

Ortega, J. L.; Aguillo, I. F. "Exploring cultural uses of an online social network: the case of Flickr". En: Journal of information sciences (en prensa).

Pissard, N.; Prieur, C. "Thematic vs. social networks in web 2.0 communities: a case study on Flickr groups", En: 9ème Rencontres francophones sur les aspects algorithmiques des telecommunications, 2007, pp. 31-34. Ile d'Oléron, France.

Prieur, C.; Cardon, D.; Beuscart, J. S.; Pissard, N.; Pons, P. The strength of weak cooperation: a case study on Flickr, 2008. Consultado en: 27-0508 .

http://arxiv.org/abs/0802.2317

Rattenbury, T.; Good, N.; Naaman, M. "Towards automatic extraction of event and place semantics from Flickr tags". En: Kraaij, W.; de Vries, A. P.; Clarke, C. L. A.; Fuhr, N.; Kando, N. (eds.). Proceedings of the thirtieth international ACM sigir conference (Sigir 2007), 2007, pp. 103-110. Amsterdam, The Netherlands: ACM Press.

Schmitz, P. "Inducing ontology from Flickr tags". En: Carr, L.; De Roure, D.; Iyengar, A.; Goble, C. A.; Dahlin, M. (eds.). Proceedings of the 15th international conference on world wide web, WWW2006, 2006. Edinburgh, UK: ACM Press.

Seidman, S. B. "Network structure and minimum degree". En: Social networks, 1983 , v. 5, pp. 269-287.

Thelwall, M. "Social networks, gender and friending: an analysis of MySpace member profiles". En: Journal of the American Society for Information Science and Technology (en prensa).

http://www.scit.wlv.ac.uk/ cm1993/papers/MySpace_preprint.doc

Torres-Salinas，D.; Cabezas-Clavijo，A.; Delgado-López-Cózar， E. "Análisis métrico de los blogs españoles de biblioteconomía y documentación (2006-2007)“. En: El profesional de la información, 2008, v. 17, n. 1 , pp. 38-48

Voss, J. “Tagging, folksonomy \& co-renaissance of manual indexing?” En: 10th International symposium of information science, 2007, pp. 234-254. Cologne, Germany.

Watts, D. J.; Strogatz, S. H. "Collective dynamics of 'small-world' networks”. En: Nature, 1998, v. 393, pp. 440-442.

José-Luis Ortega, Scientific Programming Division, Vice-presidency for Science and Technology, CSIC.

Serrano 113, 28002 Madrid.

Tel.: +34- 915855272

jortega@orgc.csic.es

Isidro-F. Aguillo, Cybermetrics Lab., Centro de Ciencias Humanas y Sociales (CCHS), CSIC.

Albasanz 26-28, 28037 Madrid.

Tel.: +34-916022 890; fax: 916022971

isidro.aguillo@cchs.csic.es

\section{Suscripciones}

Renovar (o comenzar) la suscripción a "El profesional de la información" es ágil y sencillo.

Usted puede gestionar online su suscripción conectándose a esta web:

http://www.elprofesionaldelainformacion.com/suscripciones.html

Si lo desea puede comunicar con nosotros dirigiéndose a:

suscripciones@elprofesionaldelainformacion.com

o al teléfono: +34-609352954 